\title{
Cell-type-specific DNA methylation analysis of the frontal cortices of mutant Polg 1 transgenic mice with neuronal accumulation of deleted mitochondrial DNA
}

Hiroko Sugawara ${ }^{1,2 \dagger}{ }^{+}$, Miki Bundo ${ }^{3 \dagger}$, Takaoki Kasahara ${ }^{4}$, Yutaka Nakachi $^{3}$, Junko Ueda ${ }^{3}$, Mie Kubota-Sakashita ${ }^{5}$, Kazuya Iwamoto ${ }^{3^{*}}$ and Tadafumi Kato ${ }^{5^{*}}$

\begin{abstract}
Bipolar disorder (BD) is a severe psychiatric disorder characterized by repeated conflicting manic and depressive states. In addition to genetic factors, complex gene-environment interactions, which alter the epigenetic status in the brain, contribute to the etiology and pathophysiology of BD. Here, we performed a promoter-wide DNA methylation analysis of neurons and nonneurons derived from the frontal cortices of mutant Polg1 transgenic $(n=6)$ and wild-type mice $(n=6)$. The mutant mice expressed a proofreading-deficient mitochondrial DNA (mtDNA) polymerase under the neuron-specific CamK2a promoter and showed BD-like behavioral abnormalities, such as activity changes and altered circadian rhythms. We identified a total of 469 differentially methylated regions (DMRs), consisting of 267 neuronal and 202 nonneuronal DMRs. Gene ontology analysis of DMR-associated genes showed that cell cycle-, cell division-, and inhibition of peptide activity-related genes were enriched in neurons, whereas synapse- and GABArelated genes were enriched in nonneurons. Among the DMR-associated genes, Trim2 and Lrpprc showed an inverse relationship between DNA methylation and gene expression status. In addition, we observed that mutant Polg1 transgenic mice shared several features of DNA methylation changes in postmortem brains of patients with BD, such as dominant hypomethylation changes in neurons, which include hypomethylation of the molecular motor gene and altered DNA methylation of synapse-related genes in nonneurons. Taken together, the DMRs identified in this study will contribute to understanding the pathophysiology of BD from an epigenetic perspective.
\end{abstract}

Keywords: Bipolar disorder, Schizophrenia, DNA methylation, Neuron, Nonneuron, Mitochondrial dysfunction

*Correspondence: iwamotok@kumamoto-u.ac.jp; tadafumi.kato@juntendo. ac.jp

${ }^{\dagger}$ Hiroko Sugawara and Miki Bundo contributed equally to this work.

${ }^{3}$ Department of Molecular Brain Science, Graduate School of Medical Sciences, Kumamoto University, 1-1-1 Honjo, Chuo-ku, Kumamoto-shi, Kumamoto 860-8556, Japan

${ }^{5}$ Department of Psychiatry and Behavior Science, Graduate

School of Medicine, Juntendo University, 3-1-3 Hongo, Bunkyo-ku,

Tokyo 113-8431, Japan

Full list of author information is available at the end of the article

\section{Background}

Bipolar disorder (BD) is a severe psychiatric disorder characterized by repeated conflicting states of mania and depression. A recent genome-wide association study revealed that $\mathrm{BD}$ is a polygenic disorder caused by multiple genetic risks with small effect sizes [1]. In addition to genetic factors, complex gene-environment interactions may contribute to the high heritability of BD. Epigenetics, such as DNA methylation, is the study of genetic regulation of gene expression without changing the DNA sequences [2]. The majority of DNA 
methylation occurs at the position of cytosine-guanine $(\mathrm{CpG})$ dinucleotides sequence in mammals. Usually, the extent of methylation at CpG islands, which are located within and around the regulatory promoter regions of genes, correlates with the extent of gene expression. DNA methylation, which is affected by environmental factor, have been considered to reflect gene-environment interactions and contribute to long-lasting alteration of gene expression [3, 4]. A number of DNA methylation studies have suggested to play important roles in the etiology of psychiatric disorders $[5,6]$.

Chronic progressive external ophthalmoplegia, a mitochondrial disease caused by mutations in POLG1 (DNA polymerase subunit gamma 1) and other genes encoding mitochondrial proteins, often present with comorbid mood disorders [7]. The pathogenic mutations in POLG1 generally result in the loss of proofreading and/or polymerase activities of mitochondrial DNA (mtDNA) polymerase and cause accumulation of deleted mtDNA in the cells $[8,9]$. We have previously generated transgenic $(\mathrm{Tg})$ mice carrying the mutant Polg1 (D181A) under the neuron-specific Camk2a promoter (Fig. 1a) [10]. The mutant mice showed neuron-specific accumulation of deleted mtDNA and behavioral abnormalities potentially capturing some facet of $\mathrm{BD}$, such as recurrent and spontaneous depression-like episodes associated with emotional, vegetative and psychomotor disturbances, and response to antidepressant, mood stabilizer, and electroconvulsive treatments $[8,10,11]$. In this study, we identified differentially methylated regions (DMRs) in the brains of mutant Polg1 Tg mice and identified candidate DMRs associated with the pathophysiology of BD.

\section{Methods}

We performed promoter-wide DNA methylation analysis of the isolated $\mathrm{NeuN}+$ and $\mathrm{NeuN}$ - nuclei fraction [12] derived from the frontal cortices of six $\mathrm{Tg}$ mice [male $(n=3)$ and female $(n=3)$ ] and six of their wildtype, sex-matched littermates. We used the same regions with previously performed gene expression analysis of Tg mice [13]. Cell type-specific analysis would be important in the brain tissue [12] and in this Tg mice, as the transgene specifically expresses in neurons. After collecting the densely methylated regions using $\mathrm{MBD} 2 \mathrm{~B} / 3 \mathrm{~L}$, DNA methylation profiles were obtained with the tiling arrays covering approximately 28,000 mouse promoters (Affymetrix, Santa Clara, CA, USA). DMRs were identified using model-based analysis of tiling array software [14] by comparing Tg mice and wild-type mice datasets. Detailed methods are described in the Additional file 2: Methods section.

\section{Results and discussion}

We identified a total of 469 DMRs, consisting of 267 neuronal and 202 nonneuronal DMRs. Among them, $67.4 \%(\mathrm{n}=180)$ and $41.1 \%(\mathrm{n}=83)$ were hypomethylated in neurons and nonneurons, respectively (Fig. 1b, Additional file 3: Tables S1-S4). DMRs were distributed throughout the mouse genome (Fig. 1c). Notably, in nonneurons, three DMRs detected in the upstream sequence of $\mathrm{CamK2a}$ showed hypermethylation in $\mathrm{Tg}$ mice compared to nonneurons of wild-type mice (Fig. 1a). In addition, one DMR detected in the Arsi showed hypermethylation in both cell types of Tg mice. However, these hypermethylation sites were attributed to changes in the methylation profile of the transgene construct consisting of a long upstream region of the $C a m k 2 a$ gene, which contains a part of a neighboring gene, Arsi. The fact that the promoter region of $C a m k 2 a$ on the transgene was densely methylated in only nonneurons suggested the successful neuron-specific regulation of the mutant Polg1 expression in $\mathrm{Tg}$ mice.

There were a few overlapped DMR-associated genes between neurons and nonneurons, and most of them showed cell-type-specific DNA methylation changes (Fig. 1b, Additional file 1: Discussion). Given that the deleted mtDNA primarily accumulates in neurons [10], the DMRs in nonneurons were considered to be secondarily induced by neuron-nonneuron interactions during development. The top hit list of DMR-associated genes in neurons included hypomethylation of Kif $3 b$ and hypermethylation of Sun5, Dclre1c, Bpifb2, Thoc3, and Nkain4. In nonneurons, hypomethylation of Zfp 712, Gal3st1, and Ermp1, and hypermethylation of Kcnh5 and Gipc2 were included (Fig. 1d).

We performed Gene Ontology analysis using DMRassociated genes. In neurons, we found that cell cycle-, cell division-, and inhibition of peptidase activity-related genes were enriched, whereas cell junction, synapserelated genes, and GABA receptor-related genes were enriched in nonneurons (Fig. 1c and Additional file 3: Table S5).

We then compared the DMR-associated genes and differentially expressed genes (DEGs) that were previously obtained from the frontal cortices of mutant Polg1 $\mathrm{Tg}$ mice [13]. There were seven common genes between neuronal DMR-associated genes and DEGs (Bnip2, Mylip, Psmc4, Raf1, Trim2, Galnt2, and Lrpprc) (Fig. 1e and Additional file 1: Discussion). The relatively small number of overlapping may be because gene expression profiles were obtained from unsorted bulk cortical tissues. Among the overlapping genes, two genes (Trim2 in neurons and Lrpprc in nonneurons) showed a typical relationship between hypermethylation and downregulation of gene expression (Additional file 1: Discussion). 


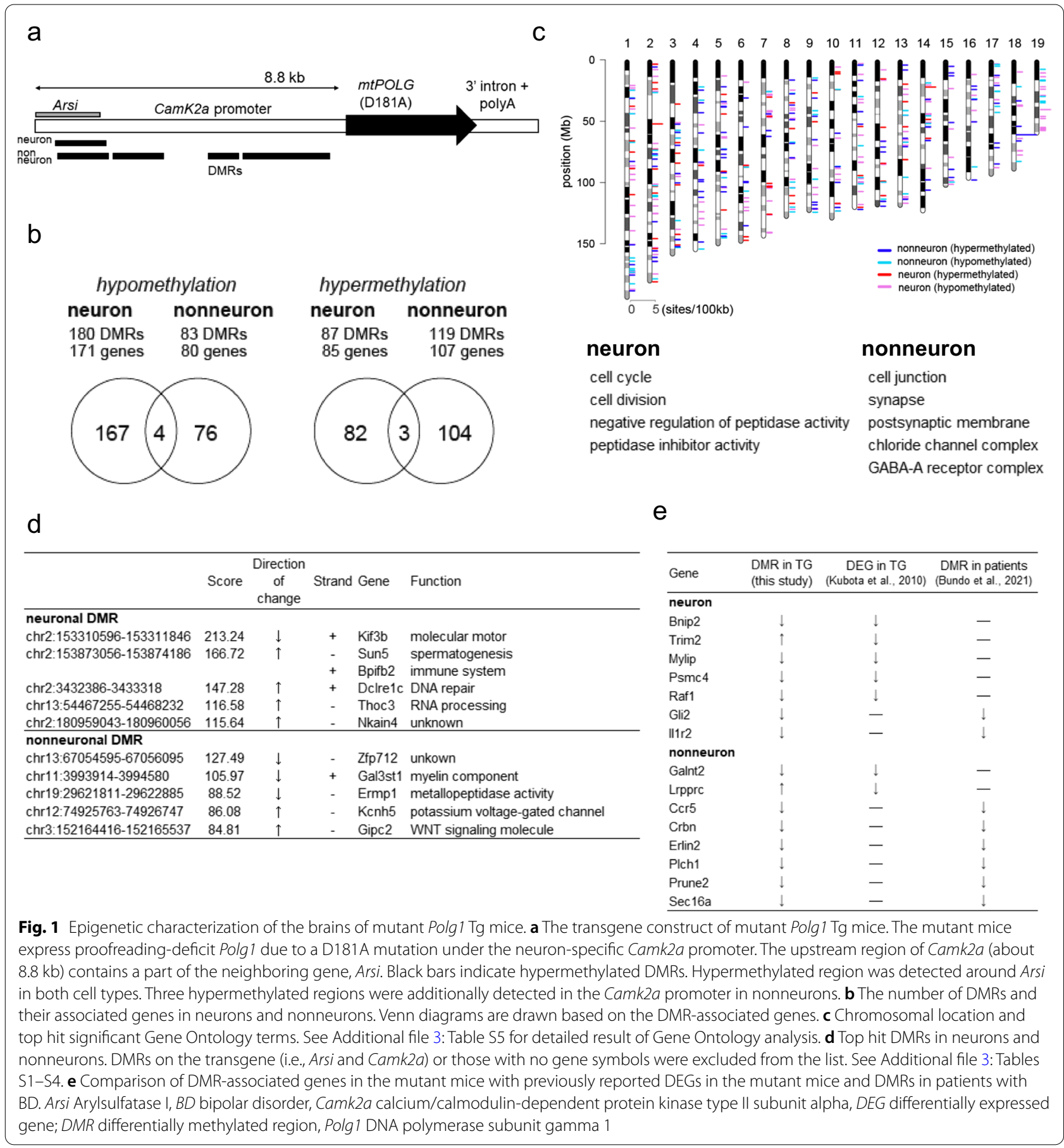

We then compared DMR-associated genes in mice and postmortem brains of patients with BD [15]. We found hypomethylation of two genes (Gli2 and Il1r2) in neurons and six genes (Ccr5, Crbn, Erlin2, Plch1, Prune2, and Sec16A) in nonneurons (Fig. 1e, Additional file 1: Discussion). We observed that DNA methylation changes in mutant Polg1 Tg mice shared several features of those identified in the postmortem brains of patients with BD. First, neurons showed more hypomethylation changes than hypermethylation changes in the mutant mice (binominal test, $\mathrm{P}<0.0001$ ) and also showed hypomethylation of molecular motor genes in neurons. Second, DMR-associated genes in nonneurons 
showed clearer enrichment in the synapse and neurotransmitter-related genes than in neurons.

In conclusion, we characterized epigenetic alterations in the neurons and nonneurons of mutant Polg1 Tg mice. There were some sex-specific behavioral alterations in mutant Polg1 Tg mice, such as the propensity for spontaneous recurrent hypoactivity in females [11]. Therefore, DMRs may be different between male and female $\mathrm{Tg}$ mice. Although further validation and functional assays as well as sex-specific analysis will be needed, the DMRs identified in this study, part of which were shared with $\mathrm{BD}$ patients, will contribute to the understanding of the pathophysiology of BD from an epigenetic perspective. Among the DMRs, those associated with gene expression changes, and those common to postmortem brains of BD will be particularly important candidates related to the pathophysiology of BD.

\section{Abbreviations}

BD: Bipolar disorder; DEG: Differentially expressed gene; DMR: Differentially methylated region; mtDNA: Mitochondrial DNA; Tg mice: Transgenic mice.

\section{Supplementary Information}

The online version contains supplementary material available at https://doi. org/10.1186/s13041-021-00894-4.

Additional file 1: Supplementary Discussion.

Additional file 2: Supplementary Methods.

Additional file 3: Supplementary Tables.

Acknowledgements

We are indebted to the Research Resource Center at RIKEN for nuclear sorting and microarray analyses.

\section{Authors' contributions}

HS and MB performed the experiments. HS, JU, YN, MK-S, and KI performed the data analysis. HS and KI wrote the manuscript. TKa, KI and TKato designed the study. All authors read and approved the final manuscript.

\section{Funding}

This research was partly supported by JSPS KAKENHI under Grant numbers: 17H01573, 18H05435, 18H05428, 18H02753, 18H05430, 18K15486, 21 K07548, and $21 \mathrm{~K} 07528$. This research was also partly supported by AMED under Grant numbers JP15gm0510002, 21wm0425006h0001, JP20dm0107123, and JP21dm0207074.

\section{Availability of data and materials}

Array data has been deposited in the Gene Expression Omnibus as GSE171120. Other datasets used and/or analyzed during the current study are available from the corresponding author on reasonable request.

\section{Declarations}

Ethics approval and consent to participate

All experimental procedures involving animals were approved by the RIKEN Center for Brain Science (CBS) Animal Committee.

\section{Consent for publication}

Not applicable.

\section{Competing interests}

The authors declare that they have no competing interests.

\section{Author details}

${ }^{1}$ Department of Psychiatry, Kansai Rosai Hospital, Amagasaki, Japan. ${ }^{2}$ Department of Psychiatry, Graduate School of Medicine, Osaka University, Osaka, Japan. ${ }^{3}$ Department of Molecular Brain Science, Graduate School of Medical Sciences, Kumamoto University, 1-1-1 Honjo, Chuo-ku, Kumamoto-shi, Kumamoto 860-8556, Japan. ${ }^{4}$ Career Development Program, RIKEN Center for Brain Science, Saitama, Japan. ${ }^{5}$ Department of Psychiatry and Behavior Science, Graduate School of Medicine, Juntendo University, 3-1-3 Hongo, Bunkyo-ku, Tokyo 113-8431, Japan.

Received: 4 November 2021 Accepted: 25 December 2021

Published online: 06 January 2022

\section{References}

1. Mullins N, Forstner AJ, O'Connell KS, Coombes B, Coleman JRI, Qiao Z, et al. Genome-wide association study of more than 40,000 bipolar disorder cases provides new insights into the underlying biology. Nat Genet. 2021;53(6):817-29.

2. Allis $C D$, Jenuwein $T$. The molecular hallmarks of epigenetic control. Nat Rev Genet. 2016;17(8):487-500.

3. Feinberg AP. Phenotypic plasticity and the epigenetics of human disease. Nature. 2007;447(7143):433-40.

4. Petronis A. Epigenetics as a unifying principle in the aetiology of complex traits and diseases. Nature. 2010;465(7299):721-7.

5. Fries GR, Li Q, McAlpin B, Rein T, Walss-Bass C, Soares JC, et al. The role of DNA methylation in the pathophysiology and treatment of bipolar disorder. Neurosci Biobehav Rev. 2016;68:474-88.

6. Nishioka M, Bundo M, Kasai K, Iwamoto K. DNA methylation in schizophrenia: progress and challenges of epigenetic studies. Genome Med. 2012;4(12):96.

7. Smits BW, Fermont J, Delnooz CC, Kalkman JS, Bleijenberg G, van Engelen BG. Disease impact in chronic progressive external ophthalmoplegia: more than meets the eye. Neuromuscul Disord. 2011;21(4):272-8.

8. Kasahara T, Ishiwata M, Kakiuchi C, Fuke S, Iwata N, Ozaki N, et al. Enrichment of deleterious variants of mitochondrial DNA polymerase gene (POLG1) in bipolar disorder. Psychiatry Clin Neurosci. 2017;71(8):518-29.

9. Longley MJ, Graziewicz MA, Bienstock RJ, Copeland WC. Consequences of mutations in human DNA polymerase gamma. Gene. 2005;354:125-31.

10. Kasahara T, Kubota M, Miyauchi T, Noda Y, Mouri A, Nabeshima T, et al. Mice with neuron-specific accumulation of mitochondrial DNA mutations show mood disorder-like phenotypes. Mol Psychiatry. 2006;11(6):577-93.

11. Kasahara T, Takata A, Kato TM, Kubota-Sakashita M, Sawada T, Kakita A et al. Depression-like episodes in mice harboring mtDNA deletions in paraventricular thalamus. Mol Psychiatry. 2016;21(1):39-48.

12. Iwamoto K, Bundo M, Ueda J, Oldham MC, Ukai W, Hashimoto E, et al. Neurons show distinctive DNA methylation profile and higher interindividual variations compared with non-neurons. Genome Res. 2011;21(5):688-96.

13. Kubota M, Kasahara T, Iwamoto K, Komori A, Ishiwata M, Miyauchi T, et al. Therapeutic implications of down-regulation of cyclophilin D in bipolar disorder. Int J Neuropsychopharmacol. 2010;13(10):1355-68.

14. Johnson WE, Li W, Meyer CA, Gottardo R, Carroll JS, Brown M, et al. Modelbased analysis of tiling-arrays for ChIP-chip. Proc Natl Acad Sci U S A. 2006;103(33):12457-62.

15. Bundo M, Ueda J, Nakachi Y, Kasai K, Kato T, Iwamoto K. Decreased DNA methylation at promoters and gene-specific neuronal hypermethylation in the prefrontal cortex of patients with bipolar disorder. Mol Psychiatry. $2021 ; 73: 526$

\section{Publisher's Note}

Springer Nature remains neutral with regard to jurisdictional claims in published maps and institutional affiliations. 